\title{
Non-linear sweep voltammetry of adsorbed species: Theory and a method to determine formal potentials
}

\author{
Enno Kätelhön and Richard G. Compton* \\ Department of Chemistry, Physical and Theoretical Chemistry Laboratory, Oxford University \\ South Parks Road, Oxford, OX1 3QZ, United Kingdom
}

\begin{abstract}
Recent literature revealed the hitherto unexploited opportunities offered by unconventional cyclic voltammetry with non-triangular potential sweeps. We here investigate the implications of such techniques for the equilibrium voltammetry of surface-bound analytes and expose rather counterintuitive effects: If only slightly different potential waveforms are applied, distinct and characteristic features arise in the voltammogram that can be readily exploited for quantitative analysis. Our work comprises a theoretical analysis and suggests initially a simple method to determine formal potentials.
\end{abstract}

\section{Introduction}

For decades electrochemical- and electroanalytical research has utilised cyclic voltammetry ${ }^{1-5}$ as the key experimental technique, which is not at least due to the simplicity of the measurement set up and procedure: An electrode surface is set in contact with a solution and its potential is varied with respect to the solution potential that remains fixed via an appropriate reference electrode. A plot of the electrode current as a function of the applied potential then yields the common representation of electrochemical data in the form of a voltammogram, which is essential and characteristic to most studies published in this field of research.

Since the rise of linear sweep cyclic voltammetry in the 1960s, a large number of alternative voltammetry methods have been introduced. Most prominently, the techniques impedance spectroscopy, staircase voltammetry, squarewave voltammetry, and differential pulse voltammetry are used frequently. All of these bear distinct advantages and disadvantages, and discussions of various methods can be found in major textbooks ${ }^{3 ; 4 ; 6}$ or review papers ${ }^{5}$. Notable techniques for the investigation of surface bound analytes further include $\mathrm{AC}$ voltammetry ${ }^{7}$ and the more recent Fourier transform large-amplitude $\mathrm{AC}$ voltammetry $^{8}$, which offer some opportunities but require a significantly more complex analysis of the experimental data than for instance standard cyclic voltammetry or the approach discussed below.

In spite of the measurement's simplicity in standard cyclic voltammetry, the nature of voltammograms is often intricate and their readability complicated. Though not always exploited to the full extent ${ }^{9}$, the comparison with theory can be of great help, if not mandatory, and aid the extraction of useful information from experiments. Paired with a rigorous analysis, voltammograms may provide insight into physiochemical properties such as prevalent reaction mechanisms, more general interfacial processes including adsorption, analyte concentrations, and diffusion coefficients. In this regard, the advantage of 
triangular sweep voltammetry particularly lies in the vast amount of theoretical legacy that has been achieved since the sixties and is today readily available to every researcher.

Often outweighed by the above advantages of simplicity and theoretical knowledge, triangular cyclic voltammetry gives rise to challenges that are specific to the shape of the applied potential waveform. Since the capacitive contribution to the measured current $I_{c}$ is proportional to the temporal gradient of the applied potential $\partial_{t} E(t), I_{c}$ is discontinuous whenever the triangular wave inflects its sweep direction. As any experimental system is necessarily affected by parasitic capacitances and other inaccuracies in the current measurement, such discontinuities inevitably generate artefacts in the experimental data. At the expense of less theory being available, this issue can be avoided if different potential waves are chosen in a way that $\partial_{t} E(t)$ and hence $I_{c}$ is continuous. Such voltammetry ${ }^{10}$ was recently investigated by Uchida et al., who analysed waveforms based on cosine functions and pointed towards exciting perspectives of novel voltammetric techniques to for instance determine formal potentials at macroelectrodes.

The following guides through the theoretical treatment of the reversible one-electron-transfer reactions of an analyte bound at an electrode surface and investigates effects arising in non-triangular sweep voltammetry. To this end, we revisit the equilibrium voltammetry of adsorbed species and the cosinebased approach to cyclic voltammetry as recently suggested ${ }^{10}$ by Uchida et al., investigate the related current dependency on the scan rate, and provide insight into the implications that are specific to this alternative approach when applied to surface-bound species. Finally, we reveal a simple alternative approach to the determination of formal potentials.

\section{Equilibrium voltammetry of surface-bound species}

The theory presented in this section is derived along the lines of a previous ${ }^{11}$ analysis, which is concerned with the triangular sweep cyclic voltammetry of adsorbed species. Various concepts may though be directly transferred to other potential waveforms as demonstrated below.

We investigate the heterogeneous reaction of two species $A$ and $B$ in a one-electron-transfer process at an electrode surface:

$$
\begin{gathered}
A_{a d s}+e \rightleftarrows B_{a d s} \\
\frac{\Gamma_{A}}{\Gamma_{B}}=\exp \left(-\frac{F}{R T}\left(E-E_{f}^{0}\right)\right)
\end{gathered}
$$

with $F, R, T$, and $E_{f}^{0}$ having their usual significance. Since as a result of the equilibrium conditions this equation does not include any time dependency, changes in the electrode potential lead to immediate adjustments of the ratio of the concentrations and Nernst equation applies throughout the entire experiment. At any point in time conservation of mass additionally demands:

$$
\Gamma_{A}+\Gamma_{B}=\Gamma_{t o t}
$$

where $\Gamma_{\text {tot }}$ is a constant noting the total amount of electro-active material $A$ and $B$ bound at the surface. The combination of (2) and (3) immediately yields:

$$
\Theta_{B}=\frac{\Gamma_{B}}{\Gamma_{t o t}}=\frac{1}{1+\exp (-\theta)}
$$


where $\Theta_{B}$ is the dimensionless fractional surface coverage of $B$ and, for mostly practical reasons, the electrode potential is written in terms of the dimensionless potential $\theta$ :

$$
\theta=\frac{F}{R T}\left(E-E_{f}^{0}\right)
$$

This provides an expression for the electrode current $I$ :

$$
I=F A \Gamma_{t o t} \cdot \frac{\partial \Theta_{B}}{\partial t}
$$

where $A$ is the area of the electrode surface. It is herein noted that temporal evolution of the electrode current is solely dependent on the applied electrode potential $E(t)$ and (6) is hence independent of the applied potential wave. For simplicity, the following writes Equation (6) in terms of the dimensionless time:

$$
\tau=\frac{t}{t_{\max }}
$$

where $t_{\max }$ is the duration of the experiment and the dimensionless flux $J$ :

$$
J=\frac{t_{\max }}{F A \Gamma_{t o t}} I
$$

Equation (6) can then simply be expressed as:

$$
J=\frac{\partial \Theta_{B}}{\partial \tau}
$$

The below voltammograms are numerically determined through Equation (9), while all calculations use the programming language Julia.

\section{Cycling voltammetry with cosine waveforms}

In a recent publication ${ }^{10}$, Uchida et al. discussed the voltammetry of a reversible electrode reaction when a potential waveform based on a cosine function is used:

$$
\theta(\tau)=A_{0} \cdot|\cos (2 \pi \tau)|^{n} \cdot \frac{\cos (2 \pi \tau)}{|\cos (2 \pi \tau)|}+\Delta \theta
$$

where $\Delta \theta$ is defined along the lines of Equation (5):

$$
\Delta \theta=\frac{F}{R T}\left(\Delta E-E_{f}^{0}\right)
$$

and where $A_{0}, n \in \mathbb{R}$ and $\Delta \theta$ is the dimensionless offset potential of the applied potential wave with respect to the formal potential at $\theta=0$, i.e. the difference between the potential around which the wave is 'centred' or mathematically:

$$
\Delta \theta=\overline{\theta(\tau)}=\int d \tau \theta(\tau)
$$

and analogously in dimensional coordinates: 

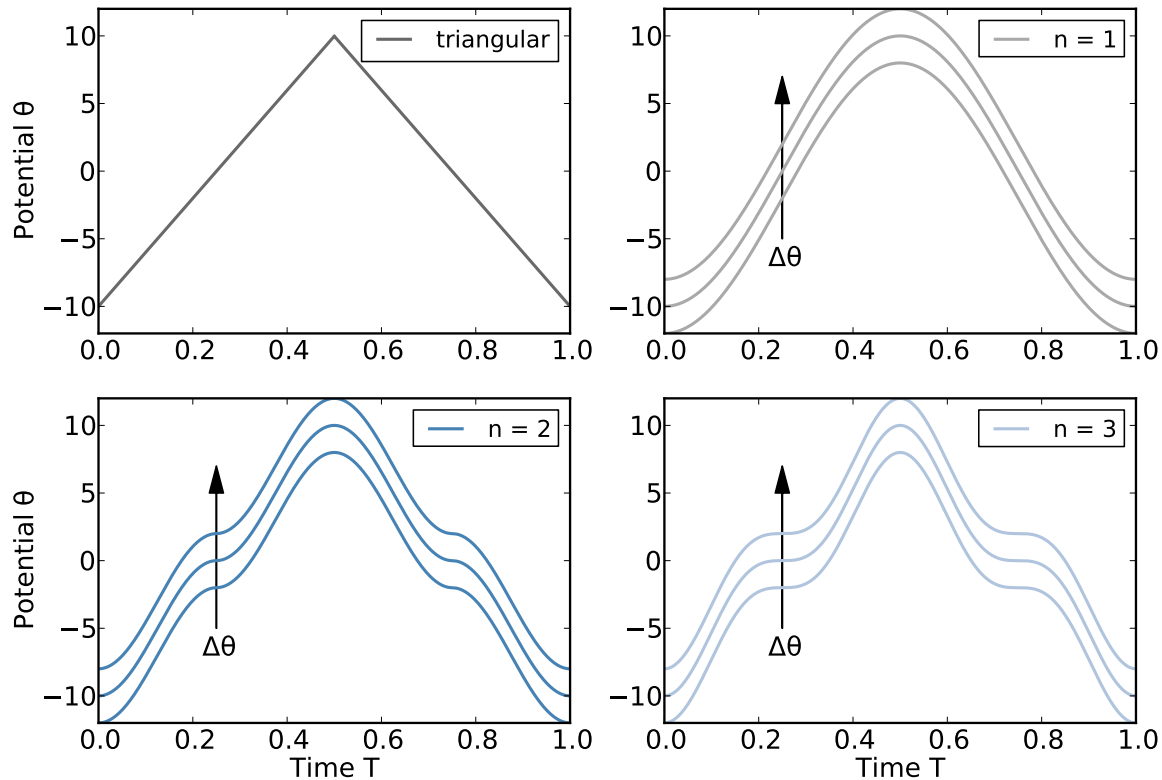

Figure 1: Triangular and alternative potential waveforms based on a cosine function as given in Equation 10. The latter is evaluated for different exponents $n=1,2,3$ and different $\Delta \theta=-2,0,2$. Please note that here and in the following plots, the amplitude $A_{0}$ is set to ten.

$$
\Delta E=\overline{E(t)}=\int d t E(t)
$$

Figure 1 depicts the shape of the wave for various offset potentials $\Delta \theta$ and exponents $n$ alongside a corresponding standard triangular wave. The figure illustrates the physical implications of the two parameters: $\Delta \theta$ shifts the waveform with respect to the reference and formal potential, while $n$ sets the extent to which the applied waveform exhibits a plateau at the potential $\Delta \theta$.

Uchida's work demonstrated that two distinct advantages arise from the use of the alternative waveform: First and most notably, distortions of the voltammetric data that result from discontinuities in the capacitive current are reduced as (10) is continuously differentiable, i.e. $\partial_{\tau} \theta$ is a continuous function, and the capacitive current contributions $I_{c} \propto \partial_{\tau} \theta$ are hence continuous as well. By this means the perturbations arising near the potentials, at which the 'classical' triangular wave inflects its sweep direction, are suppressed. Second, the formal potential $E_{f}^{0}$ of a reversible one-electron-transfer reaction at a macroelectrode can conveniently be determined by simply varying $\Delta \theta$ in a series of subsequent experiments: If $\Delta \theta$ matches $E_{f}^{0}$, a significant increase in the peak-to-peak separation is observed and immediately reveals the formal potential.

While non-triangular sweep voltammetry offers exciting advantages over more common approaches, it is though noted that the capacitive contribution to the measured current is non-constant. The exact determination of this contribution and the subtraction thereof, both generally required in all voltammetry when Faradaic currents are to be investigated, hence is at least as important as in triangular sweep voltammetry. We further point out that the interface capacitance may be a function of the applied potential, which must be considered in any experimental analysis of surface-bound analytes independent of 


\section{Scan-rate dependency of the voltammograms}

Entirely independent of the shape of a potential waveform, the above theory enables immediate insight into the scan-rate dependency of the voltammetric current. To this end, we define the scan rate $\sigma$ averaged over the potential cycle for all waveforms along the lines of triangular sweep voltammetry by keeping the wave duration $t_{\max }$ and its amplitude $A_{0}$ constant. Comparison with Figure 1 then reveals that:

$$
\sigma=\frac{\partial}{\partial \tau} \theta_{\wedge}(\tau)=4 A_{0}
$$

where the index $\wedge$ indicates a triangular waveform and which using the transformations (5) and (8) yields the dimensional average scan rate:

$$
v=\frac{R T}{F} \frac{4 A_{0}}{t_{\max }}
$$

in units of [potential $\cdot$ time $\left.^{-1}\right]$. The latter enables re-writing Equation (8) in terms of $v$ :

$$
I=\frac{F^{2} A \Gamma_{t o t} v}{4 R T A_{0}} J
$$

Counter-intuitively, the measured current is hence always proportional to the scan rate $v$. This finding is entirely independent of the shape of the potential waveform $\theta(T)$ and generally applies to all voltammetric techniques if the analyte is surface bound and Nernstian equilibrium prevails at any time.

\section{Implications for the voltammetry of surface-bound species}

The cosine-based potential waveform (10) applied to the electrode gives rise to voltammograms that differ significantly from the ones found in standard triangular sweep voltammetry: While in conventional cyclic voltammetry a single peak ${ }^{3}$ is seen, we here find two distinct peaks that drastically vary in relative magnitude as $\Delta \theta$ is shifted, see Figure 2. In contrast, the triangular wave, which generally provides the same result independent of any potential offset, exhibits a much smoother curve with, importantly, less distinct features that can be used for analysis. Due to the symmetry of the theoretical models with respect to the exchange of $A$ and $B$ all voltammograms are further symmetric with respect to the x-axis.

The above observation of the split peaks can be understood via the analysis of the applied potential: As $\theta(\tau)$ features plateaus at $\Delta \theta, \partial_{\tau} \theta(\tau)$, the temporal derivative of the surface coverage $\partial_{\tau} \Theta_{B}(\tau)$, and hence the flux $J$ must equal zero at $\Delta \theta$. Further, since the scan rate $\sigma=\partial_{\tau} \theta(\tau)$ varies with time, the peaks above and below $\Delta \theta$ as well vary with $\Delta \theta$. If $\Delta \theta$ is greater than zero, i.e. $\overline{E(t)}>E_{f}^{0}$, the first peak in an upward sweep and the the second peak in a downward sweep dominate. Analogously, if $\Delta \theta$ is smaller than zero, i.e. $\overline{E(t)}<E_{f}^{0}$, the second peak in an upward sweep and the the first peak in a downward sweep dominate. If however $\Delta \theta$ equals zero, i.e. $\overline{E(t)}=E_{f}^{0}$, all peaks feature identical absolute heights that are lower than the peak currents observed for all other cases in the Figure. 

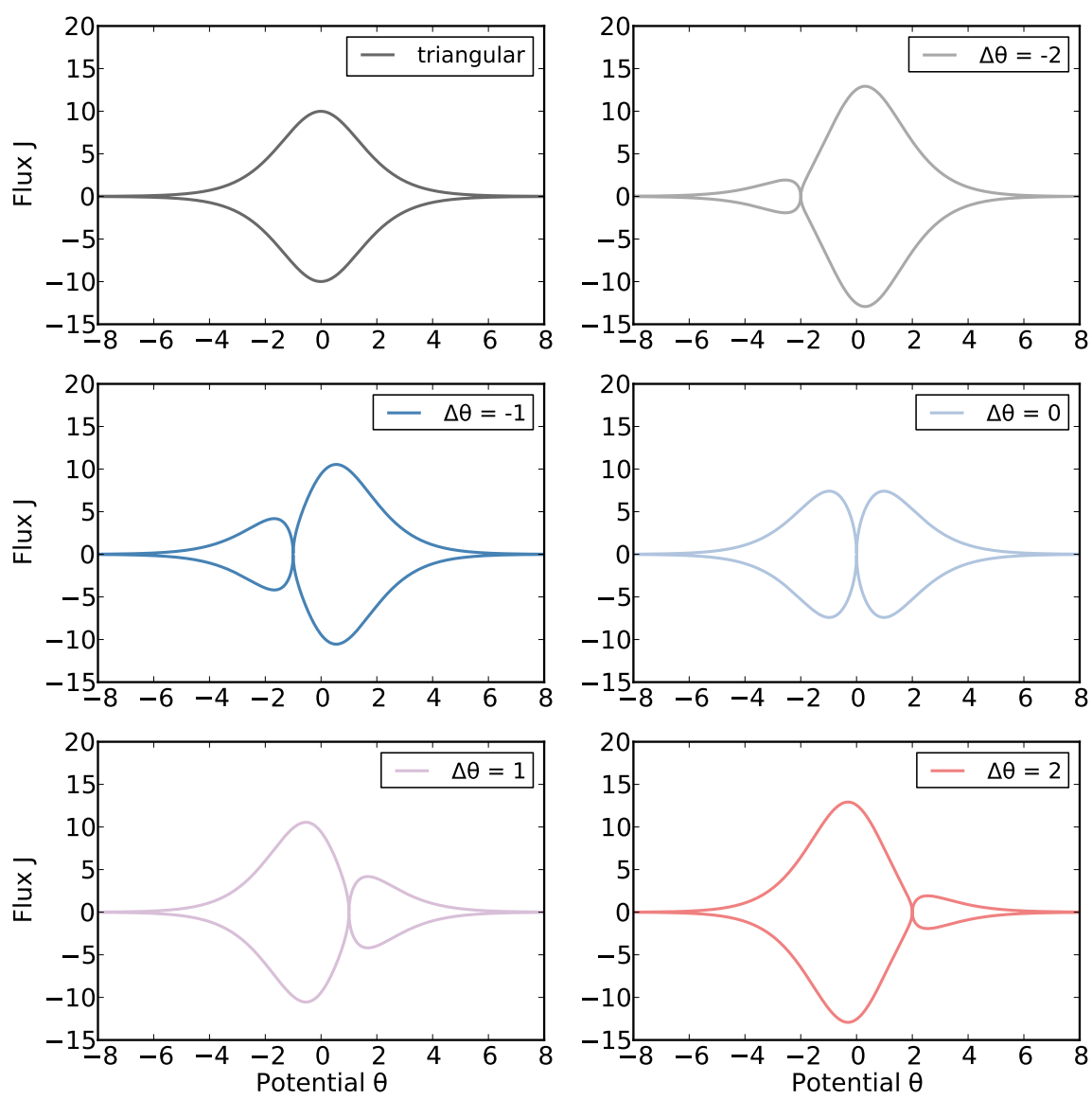

Figure 2: Numerically-found voltammograms arising from triangular- and the cosine-based potential wave (10). For the latter, an exponent $n$ of two is chosen and data is plotted for various offset potentials $\Delta \theta$ while all other parameters are held constant.

\section{Measuring formal potentials}

The previous section revealed the sensitive dependency of the voltammogram on the offset potential $\Delta \theta$ which parameterises the difference between the formal potential $E_{f}^{0}$ and the temporal average $\overline{E(t)}$ of the applied potential wave as shown in Equation (11). Most notably in this regard, we found a particularly strong influence of $\Delta \theta$ on the observed peak currents with the lowest peak currents being observed at $\Delta \theta=0$, which can be exploited to conveniently and sensibly determine a reaction formal potential.

Figure 3 plots the absolute peak current measured as a function of $\Delta \theta$ and illustrates that in the region of the formal potential around $\Delta \theta=0$, the lowest peak current is observed when $\Delta \theta=0$, i.e. the potential wave is centred at the formal potential and $\overline{E(t)}$ equals $E_{f}^{0}$. By shifting $\Delta \theta$ in a series of subsequent experiments one can hence determine the formal potential straightforwardly by simply finding the $\Delta \theta$ that corresponds to the lowest overall peak current.

For direct comparison, Figure 3 additionally plots the peak height that is obtained in standard tri- 


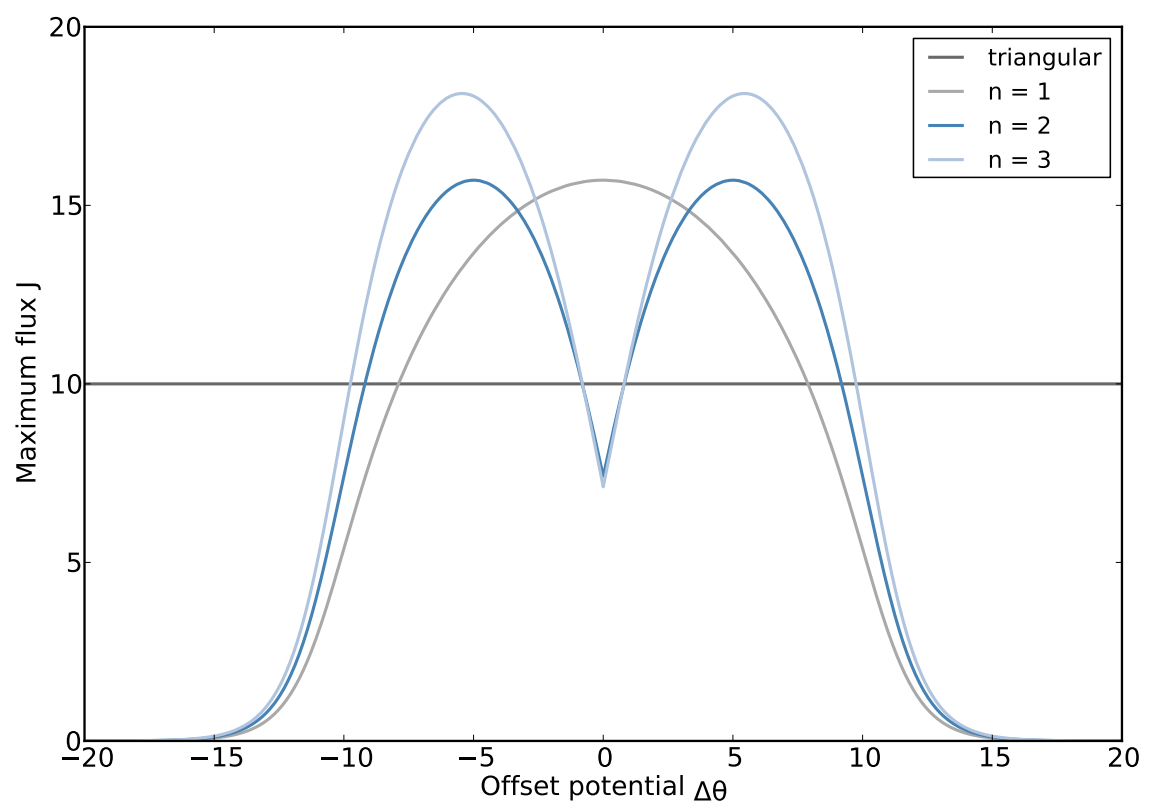

Figure 3: Maximum absolute flux $J$ arising from a cosine-based waveform as a function of the offset potential $\Delta \theta$. As in the previous figure, the exponent $n$ is set to two and the amplitude $A_{0}$ to ten. For direct comparison we additionally plot the peak current $J_{\wedge}$ observed in triangular sweep voltammetry, which is independent of a potential offset and hence appears as a vertical line in the plot.

angular cyclic voltammetry under the same conditions. The peak current for this case can be calculated analytically ${ }^{3}$ to:

$$
\max \left(I_{\wedge}\right)=\frac{F^{2}}{4 R T} v A \Gamma_{t o t}
$$

Using the transformations (5), (8), and (15) this result can be simplified to:

$$
\max \left(J_{\wedge}\right)=A_{0}
$$

[Equation was amended] which is plotted here and reflects the peak current that was numerically found ${ }^{12}$ in Figure 2. As seen in the Figure 3 and in comparison with standard cyclic voltammetry, the use of the cosine-based waveform additionally provides the advantage of much greater peak currents at the same scan rate, which particularly aids the acquisition of voltammetric data at low analyte concentrations and small electrode surfaces. The simulation of the cosine-based waveform is further much more sensitive to $E_{f}^{0}$ as the response is more complex.

\section{Conclusions}

Equilibrium voltammetry of adsorbed species with alternative potential waveforms was investigated. In vast contrast to the commonly-used triangular sweep cyclic voltammetry which yields a single broad voltammetric peak, cosine-based waveforms give rise to sharp and distinct features: Split peaks are 
observed that drastically vary in height as the offset potential is shifted respect to the formal potential. This sensitive response can be readily exploited to determine formal potentials and opens new routes that may be highly beneficial to analytical applications.

\section{Acknowledgements}

The research leading to these results has received partial funding from the European Research Council under the European Union's Seventh Framework Programme (FP/2007-2013) / ERC Grand Agreement n. [320403].

\section{References}

[1] R. S. Nicholson and I. Shain. Theory of stationary electrode polarography. single scan and cyclic methods applied to reversible, irreversible, and kinetic systems. Analytical Chemistry, 36(4):706-723, 1964.

[2] R. S. Nicholson. Theory and application of cyclic voltammetry for measurement of electrode reaction kinetics. Analytical Chemistry, 37(11):1351-1355, 1965.

[3] R. G. Compton and C. E. Banks. Understanding Voltammetry, 2nd Edition. Imperial College Press, 2011.

[4] A. J. Bard and L. R. Faulkner. Electrochemical Methods: Fundamentals and Applications, 2nd Edition. Wiley, 2001.

[5] C. Batchelor-McAuley, E. Kätelhön, E. O. Barnes, R. G. Compton, E. Laborda, and A. Molina. Recent advances in voltammetry. ChemistryOpen, 4(3):224-260, 2015.

[6] Molina, A. and González, J. Pulse voltammetry in physical electrcchemistry and electrcanalysis. Springer, 2016.

[7] Laviron, E. The use of linear potential sweep voltammetry and of ac voltammetry for the study of the surface electrochemical reaction of strongly adsorbed systems and of redox modified electrodes. Journal of Electroanalytical Chemistry and Interfacial Electrcchemistry, 100(1-2):263-270, 1979.

[8] Guo, S. and Zhang, J. and Elton, D. M. and Bond, A. M. Fourier transform large-amplitude alternating current cyclic voltammetry of surface-bound azurin. Analytical Chemistry, 76(1):166-177, 2004 .

[9] E. Kätelhön. Theory in modern electrochemistry: Science for the academic ivory tower or a vital tool for today's research? Research and Reviews in Electrochemistry, 8(1):E1-E2, 2017.

[10] Y. Uchida, E. Kätelhön, and R. G. Compton. Cyclic voltammetry with non-triangular waveforms: Electrochemically reversible systems. To be published.

[11] R. G. Compton, E. Laborda, and K. R. Ward. Understanding voltammetry: Simulation of electrode processes, $2^{\text {nd }}$ edition, chapter 7.11, page 274 ff. Imperial College Press, 2013. 
[12] E. Kätelhön and R. G. Compton. Testing and validating electroanalytical simulations. The Analyst, 140(8):2592-2598, 2015. 